DESY 00-005

hep-ph/0002071

UNIGRAZ UTP 11-01-00

January 2000

\title{
On the Structure of the Virtual Compton Amplitude in the Generalized Bjorken Region: Integral Relations
}

\author{
Johannes Blümlein ${ }^{a}$ and Dieter Robaschik ${ }^{a, b}$ \\ a Deutsche Elektronen-Synchrotron, \\ DESY - Zeuthen, Platanenallee 6, D-15735 Zeuthen, Germany \\ ${ }^{b}$ Institut für Theoretische Physik, Karl-Franzens-Universität Graz, \\ Universitätsplatz 5, A-8010 Graz, Austria
}

\begin{abstract}
A systematic study is performed of the structure of the twist-2 contributions to the virtual Compton amplitude in deep-inelastic non-forward scattering for general spin states in the non-local light cone expansion. The gauge invariance is proven in this approach for the twist-2 contributions for non-forward scattering. Relations are derived between the contributing non-forward amplitudes.
\end{abstract}




\section{Introduction}

The Compton amplitude for the scattering of a virtual photon off a hadron

$$
\gamma_{1}^{*}+p_{1} \rightarrow \gamma_{2}^{(*)^{\prime}}+p_{2}
$$

provides one of the basic tools to understand the short-distance behavior of the nucleon and to test Quantum Chromodynamics (QCD) at large space-like virtualities. In this kinematic regime the Compton amplitude is dominated by the singularities of the light-cone and can be described by the light-cone expansion [1, 2] in terms of the contributions of different twist [3]. Many investigations were devoted to the process of deep-inelastic forward scattering in the past, for which the leading, next-to-leading order, and partly the 3-loop order corrections were calculated [4]. There the absorptive part of the Compton amplitude, the hadronic tensor $W_{\mu \nu}$, is described by the four independent structure functions $F_{1}\left(x, Q^{2}\right), F_{2}\left(x, Q^{2}\right), g_{1}\left(x, Q^{2}\right)$ and $g_{2}\left(x, Q^{2}\right)$ [5].円

A more general view is obtained studying the non-forward process (1.1) without restricting it to the absorptive contributions only. In this more general kinematics a systematic description of a whole class of physical processes in the deep-inelastic region can be given and common properties of these processes can be derived. Moreover, relations obtained for forward scattering become more transparent under this generalized point of view. It is the aim of this paper to calculate the Compton amplitude for non-forward scattering in this kinematic range in lowest order in QCD. General spin states are considered which allows us to study besides the unpolarized contributions also those due to target polarization. The present analysis is devoted to the study of the twist-2 terms. The twist-decomposition of the Compton amplitude in the generalized Bjorken region is necessary since the scaling violations of the respective amplitude functions differ due to the set of the contributing operators and their anomalous dimensions [8]. The different contributions to the Compton amplitude are described by non-perturbative amplitude functions, which can be measured experimentally in the various non-forward processes. Analogously to the case of forward scattering some of the amplitude functions are experimentally easier accessible than others 2 . Therefore it is important to know the relations between the different amplitude functions due to which predictions can be made for experiment. One of the main objectives of this investigation is to derive these relations for the twist-2 parts of the amplitude functions.

The paper is organized as follows. In Section 2 the twist- 2 contributions to the Compton amplitude are calculated. We apply the non-local light-cone expansion [11, 12] to express the operator products. The non-forward expectation values are calculated for general spin states and the corresponding Lorentz-structure is derived. A helicity basis of both (virtual) photons is constructed (Section 3) which is subsequently used to determine the twist-2 contributions to the operator matrix elements. The light-cone expansion does not a priori lead to an explicit gaugeinvariant representation of the different contributions to the Compton amplitude. Since the Compton amplitude itself is gauge invariant, the expansion has to be formulated in such a way that the respective contributions under discussion form gauge-invariant sub-sets. In Section 4 we show that this is the case for the twist -2 contributions for non-forward scattering. The helicity projections of the Compton amplitude are discussed in detail in Section 5. In Section 6 we derive the relations between the twist-2 contributions to the different amplitude functions, which are

\footnotetext{
${ }^{1}$ In the case of general electroweak currents eight structure functions contribute in the massless limit, cf. [6, 7].

2 Both the measurement of the unpolarized longitudinal structure function $F_{L}\left(x, Q^{2}\right)=F_{2}\left(x, Q^{2}\right)-$ $2 x F_{1}\left(x, Q^{2}\right)$ [9] and the polarized structure function $g_{2}\left(x, Q^{2}\right)$ [10] are experimentally much more demanding than the measurement of the structure functions $F_{2}\left(x, Q^{2}\right)$ and $g_{1}\left(x, Q^{2}\right)$.
} 
generalizations of the relations known in the case of forward scattering [13, 14]. Section 7 contains the conclusions.

\section{The Compton Amplitude}

The Compton amplitude for the general case of non-forward scattering is given by

$$
T_{\mu \nu}\left(p_{+}, p_{-}, q\right)=i \int d^{4} x e^{i q x}\left\langle p_{2}, S_{2}\left|T\left(J_{\mu}(x / 2) J_{\nu}(-x / 2)\right)\right| p_{1}, S_{1}\right\rangle .
$$

Here,

$$
\begin{aligned}
& p_{+}=p_{2}+p_{1}, \quad p_{-}=p_{2}-p_{1}=q_{1}-q_{2}, \\
& q=\frac{1}{2}\left(q_{1}+q_{2}\right), \quad p_{1}+q_{1}=p_{2}+q_{2},
\end{aligned}
$$

where $q_{1}\left(q_{2}\right)$ and $p_{1}\left(p_{2}\right)$ denote the four-momenta of the incoming (outgoing) photon and hadron, respectively, and $S_{1}, S_{2}$ are the spins of the initial- and final-state hadron. Representations of the Lorentz-structure of the Compton amplitude were given in Refs. [15. In the following we will consider the Compton amplitude in the generalized Bjorken region only which is defined by the conditions

$$
\nu=q p_{+} \longrightarrow \infty, \quad-q^{2} \longrightarrow \infty
$$

keeping the variables

$$
\xi=-\frac{q^{2}}{q p_{+}}, \quad \eta=\frac{q p_{-}}{q p_{+}}=\frac{q_{1}^{2}-q_{2}^{2}}{2 \nu}
$$

fixed. The subsequent analysis will be performed demanding furthermore that the vector $q_{1}$ is spacelike and the vector $q_{2}$ can be space--, light-, or time-like, where

$$
\begin{aligned}
& q_{1}=q+\frac{1}{2} p_{-} \\
& q_{2}=q-\frac{1}{2} p_{-} .
\end{aligned}
$$

In the generalized Bjorken region the Compton amplitude is dominated by the light-cone singularities. It is therefore possible to apply the light-cone expansion for its representation. In the following we use the non-local light-cone expansion [11, 12], which is a summed-up form of the local light-cone expansion with respect to the spin indices. The respective expressions in the local light-cone expansion can be obtained from the former one by a Taylor expansion. In this way more compact representations can be obtained, cf. section 6 .

In this paper we study the twist-2 contributions to the non-forward Compton amplitude. They are obtained from the expectation values of the non-local twist-2 light cone operators, cf. [12, 16]. Here the notion of twist is used in its original form as canonical dimension - spin [3] for the local operators which are summed up to the non-local operators. In calculating the non-forward operator expectation values it turns out that the twist-decomposition performed for the operators is not necessarily complete in the case of the expectation values unlike the case for forward scattering. The emergence of new hadronic mass scales, such as $p_{+} \cdot p_{-}$or also of off-shell terms $\propto p_{+}^{2}, p_{-}^{2}$ open the possibility that expectation values of operators of lower twist mix with expectation values of operators of higher twist by virtue of these terms, cf. e.g. [17]. 
The light cone expansion in the non-forward case bears therefore additional complications to be dealt with. The impact of the hadronic mass scales $p_{+}^{2}, p_{-}^{2}$ and $p_{+} \cdot p_{-}$, which is of relevance for the contributions beyond leading twist, can in general not be dealt with as target mass- or final-state mass corrections only cf. [18, [7] for a discussion. Furthermore, the scales $p_{+}^{2}$ and $p_{-}^{2}$ dependend on the factorization of non-perturbative quantities as the distribution amplitudes and are arbitrary in this sense. In size they are comparable to the mass scales of the expectaion values of higher twist operators.

\subsection{Operator Structure}

The product of the two currents

$$
\hat{T}_{\mu \nu}(x)=i R T\left[J_{\mu}\left(\frac{x}{2}\right) J_{\nu}\left(-\frac{x}{2}\right) S\right]
$$

is then given by 3

$$
\hat{T}^{\mu \nu}(x)=-e^{2} \frac{\tilde{x}^{\lambda}}{2 \pi^{2}\left(x^{2}-i \epsilon\right)^{2}} R T\left[\bar{\psi}\left(\frac{\tilde{x}}{2}\right) \gamma^{\mu} \gamma^{\lambda} \gamma^{\nu} \psi\left(-\frac{\tilde{x}}{2}\right)-\bar{\psi}\left(-\frac{\tilde{x}}{2}\right) \gamma^{\mu} \gamma^{\lambda} \gamma^{\nu} \psi\left(\frac{\tilde{x}}{2}\right)\right] S .
$$

$\tilde{x}$ denotes a light-like vector corresponding to $x$,

$$
\tilde{x}=x+\frac{\zeta}{\zeta^{2}}\left[\sqrt{x \cdot \zeta^{2}-x^{2} \zeta^{2}}-x \cdot \zeta\right]
$$

and $\zeta$ is a subsidiary vector. The leading order expressions turn out to be independent of $\zeta$. f $e$ denotes the charge of the fermion field $\psi$, which is either a quark- or an antiquark field. $\hat{T}_{\mu \nu}(x)$, Eq. (2.9), refers therefore to the contribution of one of these fields for a single flavor. In the subsequent treatment there is no essential structural difference considering quark or antiquark fields since we work in the massless approximation. The expectation values of the bilocal quark and antiquark operators between nucleon states introduced below are of course different and do also depend on the quark flavor. The complete Compton amplitude is obtained summing Eq. (2.9) over all quark and antiquark flavors contributing in the kinematic domain considered.

The operators

$$
\bar{\psi}\left(\frac{\tilde{x}}{2}\right) \gamma^{\mu} \gamma^{\lambda} \gamma^{\nu} \psi\left(-\frac{\tilde{x}}{2}\right)
$$

are bilocal operators on the light ray $\tilde{x}$. These are renormalized and time ordered operators [12]. One may rewrite the operator $\hat{T}_{\mu \nu}(x)$ in terms of a symmetric and an asymmetric contribution by

$$
\hat{T}_{\mu \nu}(x)=-e^{2} \frac{\tilde{x}^{\lambda}}{i \pi^{2}\left(x^{2}-i \epsilon\right)^{2}}\left[S_{\alpha \mu \lambda \nu} O^{\alpha}\left(\frac{\tilde{x}}{2},-\frac{\tilde{x}}{2}\right)+i \varepsilon_{\mu \lambda \nu \sigma} O_{5}^{\alpha}\left(\frac{\tilde{x}}{2},-\frac{\tilde{x}}{2}\right)\right],
$$

where

$$
S_{\alpha \mu \lambda \nu}=g_{\alpha \mu} g_{\lambda \nu}+g_{\lambda \mu} g_{\alpha \nu}-g_{\mu \nu} g_{\lambda \alpha} .
$$

\footnotetext{
${ }^{3}$ Here and in the following, the normal product symbols for the operator products will be omitted.

${ }^{4}$ The respective terms are suppressed $\propto \sqrt{\left|\zeta^{2} / q^{2}\right|}$ and do therefore not contribute at the twist-2 level but might be of relevance for the higher twist terms beginning with twist-3.
} 
The essential objects are the bilocal light-ray operators

$$
\begin{aligned}
O^{\alpha}\left(\frac{\tilde{x}}{2},-\frac{\tilde{x}}{2}\right) & =\frac{i}{2}\left[\bar{\psi}\left(\frac{\tilde{x}}{2}\right) \gamma^{\alpha} \psi\left(-\frac{\tilde{x}}{2}\right)-\bar{\psi}\left(-\frac{\tilde{x}}{2}\right) \gamma^{\alpha} \psi\left(\frac{\tilde{x}}{2}\right)\right] \\
O_{5}^{\alpha}\left(\frac{\tilde{x}}{2},-\frac{\tilde{x}}{2}\right) & =\frac{i}{2}\left[\bar{\psi}\left(\frac{\tilde{x}}{2}\right) \gamma_{5} \gamma^{\alpha} \psi\left(-\frac{\tilde{x}}{2}\right)+\bar{\psi}\left(-\frac{\tilde{x}}{2}\right) \gamma_{5} \gamma^{\alpha} \psi\left(\frac{\tilde{x}}{2}\right)\right] .
\end{aligned}
$$

These operators do still contain higher twist contributions. In the present paper we are going to discuss the twist-2 contributions only and have therefore to perform a twist decomposition. The scalar twist-2 operators on the light-cone are given by

$$
\begin{aligned}
O\left(\frac{\tilde{x}}{2},-\frac{\tilde{x}}{2}\right) & =\tilde{x}_{\alpha} O^{\alpha}\left(\frac{\tilde{x}}{2},-\frac{\tilde{x}}{2}\right) \\
O_{5}\left(\frac{\tilde{x}}{2},-\frac{\tilde{x}}{2}\right) & =\tilde{x}_{\alpha} O_{5}^{\alpha}\left(\frac{\tilde{x}}{2},-\frac{\tilde{x}}{2}\right) .
\end{aligned}
$$

Their general definition off the light-cone can be found in Refs. [16, 12. The operators satisfy the condition

$$
\square O_{(5)}^{q, \text { traceless }}(-\kappa x, \kappa x)=0 .
$$

Here $\kappa$ parametrizes the position on the $x$-ray. The twist- 2 vector operators can now be constructed referring to the scalar operator by

$$
\begin{aligned}
O_{\sigma}^{q, \text { twist } 2}(-\kappa \tilde{x}, \kappa \tilde{x}) & =\left.\int_{0}^{1} d \tau \partial_{\sigma} O_{\text {traceless }}^{q}(-\kappa \tau x, \kappa \tau x)\right|_{x \rightarrow \tilde{x}} \\
& =\left.\int_{0}^{1} d \tau\left[\partial_{\sigma}+\frac{1}{2}(\ln \tau) x_{\sigma} \square\right] O^{q}(-\kappa \tau x, \kappa \tau x)\right|_{x=\tilde{x}}
\end{aligned}
$$

These operators satisfy the relations

$$
\partial^{\sigma} O_{\sigma}^{q, \text { traceless }}(-\kappa x, \kappa x)=0, \quad \square O_{\sigma}^{q, \text { traceless }}(-\kappa x, \kappa x)=0 .
$$

\subsection{Operator Matrix Elements}

Up to now we have considered an expression for the product of the two electromagnetic currents. As the next step we form matrix elements of the respective operators. Let us first consider the matrix element of the scalar operator. We use the kinematic decomposition into a DIRACand a PAULI-type contribution, the latter of which vanishes in the case of forward scattering, $p_{-}, \eta \rightarrow 0$.

$$
\begin{aligned}
e^{2}\left\langle p_{2}, S_{2}\left|O\left(\frac{x}{2},-\frac{x}{2}\right)\right| p_{1}, S_{1}\right\rangle & =i \bar{u}\left(p_{2}, S_{2}\right) \gamma x u\left(p_{1}, S_{1}\right) \int D z e^{-i x p_{z} / 2} f\left(z_{1}, z_{2}, p_{i} p_{j} x^{2}, p_{i} p_{j}, \mu_{R}^{2}\right) \\
& +i \bar{u}\left(p_{2}, S_{2}\right) x \sigma p_{-} u\left(p_{1}, S_{1}\right) \int D z e^{-i x p_{z} / 2} g\left(z_{1}, z_{2}, p_{i} p_{j} x^{2}, p_{i} p_{j}, \mu_{R}^{2}\right)
\end{aligned}
$$

Here $\mu_{R}$ denotes the renormalization scale and

$$
D z=\frac{1}{2} d z_{1} d z_{2} \theta\left(1-z_{1}\right) \theta\left(1+z_{1}\right) \theta\left(1-z_{2}\right) \theta\left(1+z_{2}\right)
$$




$$
\begin{aligned}
& =d z_{+} d z_{-} \theta\left(1+z_{+}+z_{-}\right) \theta\left(1+z_{+}-z_{-}\right) \theta\left(1-z_{+}+z_{-}\right) \theta\left(1-z_{+}-z_{-}\right) \\
p_{z} & =p_{+} z_{+}+p_{-} z_{-} \\
z_{ \pm} & =\frac{1}{2}\left(z_{2} \pm z_{1}\right) \\
\sigma_{\alpha \beta} & =\frac{i}{2}\left[\gamma_{\alpha} \gamma_{\beta}-\gamma_{\beta} \gamma_{\alpha}\right] .
\end{aligned}
$$

We consider all hadronic mass scales $p_{i} \cdot p_{j} \approx 0$ as small compared to the large invariants $p_{ \pm} . q$ and $q^{2}$. Therefore the on-shell relations

$$
\begin{aligned}
& \gamma_{\mu} p_{1}^{\mu} u\left(p_{1}, S_{1}\right)=0 \\
& \bar{u}\left(p_{2}, S_{2}\right) \gamma_{\mu} p_{2}^{\mu}=0
\end{aligned}
$$

hold. Under these assumptions we show that the scalar matrix element satisfies the condition (2.17):

$$
\begin{aligned}
& \left.\square e^{2}\left\langle p_{2}, S_{2}\left|O\left(\frac{x}{2},-\frac{x}{2}\right)\right| p_{1}, S_{1}\right\rangle\right|_{x \rightarrow \tilde{x}} \\
& =\quad i \square\left[\bar{u}\left(p_{2}, S_{2}\right) \gamma x u\left(p_{1}, S_{1}\right) \int D z e^{-i x p_{z} / 2} f\left(z_{1}, z_{2}\right)\right. \\
& \left.\quad+\bar{u}\left(p_{2}, S_{2}\right) x \sigma p_{u}\left(p_{1}, S_{1}\right) \int D z e^{-i x p_{z} / 2} g\left(z_{1}, z_{2}\right)\right]\left.\right|_{x \rightarrow \tilde{x}} \\
& =\quad i \int D z e^{-i \tilde{x} p(z) / 2} f\left(z_{1}, z_{2}\right)\left[-\frac{i}{2} p_{z \mu}+2 \tilde{x}_{\mu}\left(-\frac{i}{2} p_{z}\right)^{2}\right] \bar{u}\left(p_{2}, S_{2}\right) \gamma^{\mu} u\left(p_{1}, S_{1}\right) \\
& \quad+\quad i \int D z e^{-i \tilde{x} p(z) / 2} f\left(z_{1}, z_{2}\right)\left[-\frac{i}{2} p_{z \mu}+2 \tilde{x}_{\mu}\left(-\frac{i}{2} p_{z}\right)^{2}\right] \bar{u}\left(p_{2}, S_{2}\right) \sigma^{\mu \nu} p_{-\nu} u\left(p_{1}, S_{1}\right) \simeq 0
\end{aligned}
$$

Analogously to the construction of the vector operator out of the scalar operator (2.18) one obtains the matrix element

$$
\begin{aligned}
e^{2}\left\langle p_{2}, S_{2}\left|O^{\mu}\left(\frac{\tilde{x}}{2},-\frac{\tilde{x}}{2}\right)\right| p_{1}, S_{1}\right\rangle= & i \int_{0}^{1} d \lambda \partial_{x}^{\mu}\left\{\bar{u}\left(p_{2}, S_{2}\right) \gamma x u\left(p_{1}, S_{1}\right) \int D z e^{-i \lambda x p_{z} / 2} f\left(z_{1}, z_{2}\right)\right. \\
& \left.+\bar{u}\left(p_{2}, S_{2}\right) x \sigma p_{-} u\left(p_{1}, S_{1}\right) \int D z e^{-i \lambda x p_{z} / 2} g\left(z_{1}, z_{2}\right)\right\}\left.\right|_{x \rightarrow \tilde{x}} \\
= & i \int D z \partial_{x}^{\mu}\left\{\bar{u}\left(p_{2}, S_{2}\right) \gamma x u\left(p_{1}, S_{1}\right) e^{-i x p_{z} / 2} \int_{0}^{1} \frac{d \lambda}{\lambda^{2}} f\left(\frac{z_{1}}{\lambda}, \frac{z_{2}}{\lambda}\right)\right. \\
& \left.+\bar{u}\left(p_{2}, S_{2}\right) x \sigma p_{-} u\left(p_{1}, S_{1}\right) e^{-i x p_{z} / 2} \int_{0}^{1} \frac{d \lambda}{\lambda^{2}} g\left(\frac{z_{1}}{\lambda}, \frac{z_{2}}{\lambda}\right)\right\}\left.\right|_{x \rightarrow \tilde{x}} .
\end{aligned}
$$

The expectation values of the operators $O^{\mu}$ and $Q_{5}^{\mu}$ are finally expressed by

$$
\begin{aligned}
& e^{2}\left\langle p_{2}, S_{2}\left|O^{\mu}\left(\frac{\tilde{x}}{2},-\frac{\tilde{x}}{2}\right)\right| p_{1}, S_{1}\right\rangle \\
& \quad=i \int D z e^{-i \tilde{x} p_{z} / 2} F\left(z_{1}, z_{2}\right)\left[\bar{u}\left(p_{2}, S_{2}\right) \gamma^{\mu} u\left(p_{1}, S_{1}\right)-\frac{i}{2} p_{z}^{\mu} \bar{u}\left(p_{2}, S_{2}\right) \gamma \tilde{x} u\left(p_{1}, S_{1}\right)\right] \\
& \quad+i \int D z e^{-i \tilde{x} p_{z} / 2} G\left(z_{1}, z_{2}\right)\left[\bar{u}\left(p_{2}, S_{2}\right) \sigma^{\mu \nu} p_{-\nu} u\left(p_{1}, S_{1}\right)-\frac{i}{2} p_{z}^{\mu} \bar{u}\left(p_{2}, S_{2}\right) \sigma^{\alpha \beta} \tilde{x}_{\alpha} p_{-\beta} u\left(p_{1}, S_{1}\right)\right]
\end{aligned}
$$


and

$$
\begin{aligned}
& e^{2}\left\langle p_{2}, S_{2}\left|O_{5}^{\mu}\left(\frac{\tilde{x}}{2},-\frac{\tilde{x}}{2}\right)\right| p_{1}, S_{1}\right\rangle \\
& \quad=i \int D z e^{-i \tilde{x} p_{z} / 2} F_{5}\left(z_{1}, z_{2}\right)\left[\bar{u}\left(p_{2}, S_{2}\right) \gamma_{5} \gamma^{\mu} u\left(p_{1}, S_{1}\right)-\frac{i}{2} p_{z}^{\mu} \bar{u}\left(p_{2}, S_{2}\right) \gamma_{5} \gamma \tilde{x} u\left(p_{1}, S_{1}\right)\right] \\
& \quad+i \int D z e^{-i \tilde{x} p_{z} / 2} G_{5}\left(z_{1}, z_{2}\right)\left[\bar{u}\left(p_{2}, S_{2}\right) \gamma_{5} \sigma^{\mu \nu} p_{-\nu} u\left(p_{1}, S_{1}\right)-\frac{i}{2} p_{z}^{\mu} \bar{u}\left(p_{2}, S_{2}\right) \gamma_{5} \sigma^{\alpha \beta} \tilde{x}_{\alpha} p_{-\beta} u\left(p_{1}, S_{1}\right)\right]
\end{aligned}
$$

respectively. The new functions $F, G \equiv H$ and $F_{5}, G_{5} \equiv H_{5}$ are defined by

$$
H_{(5)}\left(z_{1}, z_{2}\right)=\int_{0}^{1} \frac{d \lambda}{\lambda^{2}} h_{(5)}\left(\frac{z_{1}}{\lambda}, \frac{z_{2}}{\lambda}\right)
$$

with $h_{(5)} \equiv f, g, f_{5}, g_{5}$. In the same manner as for the scalar operator it can be shown that the conditions (2.19) for the vector operator are satisfied by the matrix element.

The functions $H$ and $H_{5}$ obey the relations

$$
\begin{aligned}
H\left(z_{1}, z_{2}\right) & =-H\left(-z_{1},-z_{2}\right) \\
H_{5}\left(z_{1}, z_{2}\right) & =+H_{5}\left(-z_{1},-z_{2}\right),
\end{aligned}
$$

which follow from Eqs. (2.13,2.14) interchanging $x$ and $-x$, in the limit in which contributions due to $\zeta$, Eq. (2.10), can be disregarded.

The contributions due to the above operators to the Compton amplitude read

$$
\begin{aligned}
T_{\mu \nu}\left(p_{+}, p_{-}, q\right)= & i \int d^{4} x e^{i q x}\left\langle p_{2}, S_{2}\left|T\left(J_{\mu}(x / 2) J_{\nu}(-x / 2)\right)\right| p_{1}, S_{1}\right\rangle \\
= & \int d^{4} x e^{i q x}\left\{-\frac{\tilde{x}^{\lambda}}{i \pi^{2}\left(x^{2}-i \varepsilon\right)^{2}}\left[S_{\alpha \mu \lambda \nu}\left\langle p_{2}\left|O^{\alpha}\left(\frac{\tilde{x}}{2},-\frac{\tilde{x}}{2}\right)\right| p_{1}\right\rangle\right.\right. \\
& \left.\left.+i \varepsilon_{\mu \lambda \nu \sigma}\left\langle p_{2}\left|O_{5}^{\alpha}\left(\frac{\tilde{x}}{2},-\frac{\tilde{x}}{2}\right)\right| p_{1}\right\rangle\right]\right\} .
\end{aligned}
$$

We rewrite Eq. (2.31) referring to the distribution functions $F_{(5)}, G_{(5)}$,

$$
\begin{aligned}
& T_{\mu \nu}\left(p_{+}, p_{-}, q\right)=-\int d^{4} x e^{i\left(q-p_{z} / 2\right) x} \frac{\tilde{x}^{\lambda}}{\pi^{2}\left(x^{2}-i \varepsilon\right)^{2}} \\
& \quad \times\left\{S _ { \alpha \mu \lambda \nu } \left\{\int D z\left[\bar{u}\left(p_{2}, S_{2}\right) \gamma^{\alpha} u\left(p_{1}, S_{1}\right)-\frac{i}{2} p_{z}^{\mu} \bar{u}\left(p_{2}, S_{2}\right) \gamma \tilde{x} u\left(p_{1}, S_{1}\right)\right] F\left(z_{+}, z_{-}\right)\right.\right. \\
& \left.\quad+\int D z\left[\bar{u}\left(p_{2}, S_{2}\right) \sigma^{\alpha \beta} p_{-\beta} u\left(p_{1}, S_{1}\right)-\frac{i}{2} p_{z}^{\alpha} \bar{u}\left(p_{2}, S_{2}\right) \sigma^{\beta \gamma} \tilde{x}_{\beta} p_{-\gamma} u\left(p_{1}, S_{1}\right)\right] G\left(z_{+}, z_{-}\right)\right\} \\
& \quad+i \varepsilon_{\mu \lambda \nu \sigma}\left\{\int D z\left[\bar{u}\left(p_{2}, S_{2}\right) \gamma_{5} \gamma^{\sigma} u\left(p_{1}, S_{1}\right)-\frac{i}{2} p_{z}^{\sigma} \bar{u}\left(p_{2}, S_{2}\right) \gamma_{5} \gamma \tilde{x} u\left(p_{1}, S_{1}\right)\right] F_{5}\left(z_{+}, z_{-}\right)\right. \\
& \left.\left.\quad+\int D z\left[\bar{u}\left(p_{2}, S_{2}\right) \gamma_{5} \sigma^{\sigma \alpha} p_{-\alpha} u\left(p_{1}, S_{1}\right)-\frac{i}{2} p_{z}^{\sigma} \bar{u}\left(p_{2}, S_{2}\right) \gamma_{5} \sigma^{\alpha \beta} \tilde{x}_{\alpha} p_{-\beta} u\left(p_{1}, S_{1}\right)\right] G_{5}\left(z_{+}, z_{-}\right)\right\}\right\} .
\end{aligned}
$$

Finally the Fourier transform to momentum space is performed,

$$
T_{\mu \nu}\left(q, p_{+}, p_{-}\right)=-2 \int D z \frac{1}{Q^{2}+i \varepsilon}\left\{\bar{u}\left(p_{2}, S_{2}\right) \Gamma_{\mu \nu}^{F}\left(q, p_{+}, p_{-}\right) u\left(p_{1}, S_{1}\right) F\left(z_{+}, z_{-}\right)\right.
$$




$$
\begin{aligned}
& +\bar{u}\left(p_{2}, S_{2}\right) \Gamma_{\mu \nu}^{F 5}\left(q, p_{+}, p_{-}\right) u\left(p_{1}, S_{1}\right) F_{5}\left(z_{+}, z_{-}\right) \\
& +\bar{u}\left(p_{2}, S_{2}\right) \Gamma_{\mu \nu}^{G}\left(q, p_{+}, p_{-}\right) u\left(p_{1}, S_{1}\right) G\left(z_{+}, z_{-}\right) \\
& \left.+\bar{u}\left(p_{2}, S_{2}\right) \Gamma_{\mu \nu}^{G 5}\left(q, p_{+}, p_{-}\right) u\left(p_{1}, S_{1}\right) G_{5}\left(z_{+}, z_{-}\right)\right\}
\end{aligned}
$$

The matrices $\Gamma_{\mu \nu}^{O}$ are given by

$$
\begin{aligned}
& \Gamma_{\mu \nu}^{F}\left(q, p_{z}\right)=\left[Q_{\mu} \gamma_{\nu}+Q_{\nu} \gamma_{\mu}-g_{\mu \nu} \gamma_{\alpha} Q^{\alpha}\right] \\
& -\frac{1}{2}\left[p_{z \mu} \gamma_{\nu}+p_{z \nu} \gamma_{\mu}-g_{\mu \nu} \gamma_{\alpha} p_{z}^{\alpha}\right] \\
& +\frac{1}{Q^{2}+i \varepsilon} \gamma_{\alpha} Q^{\alpha}\left[Q_{\nu} p_{z \mu}+Q_{\mu} p_{z \nu}-g_{\mu \nu} Q \cdot p_{z}\right] \\
& \simeq\left[q_{\mu} \gamma_{\nu}+q_{\nu} \gamma_{\mu}-g_{\mu \nu} \gamma_{\alpha} q^{\alpha}\right]-\left[p_{z \mu} \gamma_{\nu}+p_{z \nu} \gamma_{\mu}\right] \\
& +\frac{1}{Q^{2}+i \varepsilon} \gamma_{\alpha} q^{\alpha}\left[-p_{z \nu} p_{z \mu}+q_{\nu} p_{z \mu}+q_{\mu} p_{z \nu}-g_{\mu \nu} q . p_{z}\right] \\
& \Gamma_{\mu \nu}^{F 5}\left(q, p_{z}\right)=i \gamma_{5} \varepsilon_{\mu \nu \lambda \sigma}\left[Q^{\lambda} \gamma^{\sigma}-\frac{1}{2} p_{z}^{\sigma} \gamma^{\lambda}+\frac{1}{Q^{2}+i \varepsilon} Q^{\lambda} p_{z}^{\sigma} \gamma_{\alpha} Q^{\alpha}\right] \\
& \simeq i \gamma_{5} \varepsilon_{\mu \nu \lambda \sigma}\left[q^{\lambda} \gamma^{\sigma}+\frac{1}{Q^{2}+i \varepsilon} q^{\lambda} p_{z}^{\sigma} \gamma_{\alpha} q^{\alpha}\right] \\
& \Gamma_{\mu \nu}^{G}\left(q, p_{z}\right)=\left[Q_{\mu} \sigma_{\nu \alpha} p_{-}^{\alpha}+Q_{\nu} \sigma_{\mu \alpha} p_{-}^{\alpha}-g_{\mu \nu} \sigma_{\alpha \beta} p_{-}^{\beta} Q^{\alpha}\right] \\
& -\frac{1}{2}\left[p_{z \mu} \sigma_{\nu \alpha} p_{-}^{\alpha}+p_{z \nu} \sigma_{\mu \alpha} p_{-}^{\alpha}-g_{\mu \nu} \sigma_{\beta \alpha} p_{-}^{\alpha} p_{z}^{\beta}\right] \\
& +\frac{1}{Q^{2}+i \varepsilon} \sigma_{\beta \alpha} p_{-}^{\alpha} Q^{\beta}\left[Q_{\nu} p_{z \mu}+Q_{\mu} p_{z \nu}-g_{\mu \nu} Q . p_{z}\right] \\
& \simeq \quad\left[q_{\mu} \sigma_{\nu \alpha} p_{-}^{\alpha}+q_{\nu} \sigma_{\mu \alpha} p_{-}^{\alpha}-g_{\mu \nu} \sigma_{\beta \alpha} p_{-}^{\alpha} q^{\beta}\right]-\left[p_{z \mu} \sigma_{\nu \alpha} p_{-}^{\alpha}+p_{z \nu} \sigma_{\mu \alpha} p_{-}^{\alpha}\right] \\
& +\frac{1}{Q^{2}+i \varepsilon} \sigma_{\beta \alpha} p_{-}^{\alpha} q^{\beta}\left[-p_{z \mu} p_{z \nu}+q_{\nu} p_{z \mu}+q_{\mu} p_{z \nu}-g_{\mu \nu} q \cdot p_{z}\right] \\
& \Gamma_{\mu \nu}^{G 5}\left(q, p_{z}\right)=i \gamma_{5} \varepsilon_{\mu \nu \lambda \sigma}\left[Q^{\lambda} \sigma^{\sigma \alpha} p_{-\alpha}-\frac{1}{2} p_{z}^{\sigma} \sigma^{\lambda \alpha} p_{-\alpha}+\frac{1}{Q^{2}+i \varepsilon} Q^{\lambda} p_{z}^{\sigma} \sigma^{\alpha \beta} Q_{\alpha} p_{-\beta}\right] \\
& \simeq i \gamma_{5} \varepsilon_{\mu \nu \lambda \sigma}\left[q^{\lambda} \sigma^{\sigma \alpha} p_{-\alpha}+\frac{1}{Q^{2}+i \varepsilon} q^{\lambda} p_{z}^{\sigma} \sigma^{\alpha \beta} q_{\alpha} p_{-\beta}\right]
\end{aligned}
$$

where

$$
Q=q-\frac{p_{z}}{2}
$$

The equivalence-sign denotes that the other contributions vanish between the bi-spinor states according to the assumption that $p_{i} \cdot p_{j} \approx 0$.

\subsection{Lorentz Structure}

Let us now discuss the Lorentz structure of the Compton amplitude in more detail. The matrices $\Gamma_{\mu \nu}^{O} /\left(Q^{2}+i \varepsilon\right)$ consist out of two parts which are $\propto 1 / Q^{2}$ and $\propto 1 / Q^{4}$, respectively. The 
numerators of the former terms depend on $q$ only, whereas the second terms contain the vectors $q$ and $p_{z}=z_{+} p_{+}+z_{-} p_{-}$. The $\left(z_{+}, z_{-}\right)$-dependence may be factored out of the quantities

$$
\bar{u}\left(p_{2}, S_{2}\right) \gamma_{\mu_{1}} \ldots \gamma_{\mu_{k}}\left(\gamma_{5}\right) u\left(p_{1}, S_{1}\right)
$$

and the form factors related to the contributions $\propto 1 / Q^{2}$ and $\propto 1 / Q^{4}$ are of different structure, graded by the $z_{ \pm}$dependence in the numerators. Due to the different numerator structure individual variations in $q, p_{+}$and $p_{-}$may allow to disentangle the different contributions experimentally. The Compton amplitude is given by

$$
\begin{aligned}
T_{\mu \nu}\left(q, p_{+}, p_{-}\right)=-2 \bar{u}\left(p_{2}, S_{2}\right)\left[\Gamma_{\mu \nu}^{F}\left(q, p_{+}, p_{-}\right)+\Gamma_{\mu \nu}^{F 5}\left(q, p_{+}, p_{-}\right)\right. \\
\left.+\Gamma_{\mu \nu}^{G}\left(q, p_{+}, p_{-}\right)+\Gamma_{\mu \nu}^{G 5}\left(q, p_{+}, p_{-}\right)\right] u\left(p_{1}, S_{1}\right),
\end{aligned}
$$

with

$$
\begin{aligned}
\Gamma_{\mu \nu}^{F}\left(q, p_{+}, p_{-}\right)= & {\left[q_{\mu} \gamma_{\nu}+q_{\nu} \gamma_{\mu}-g_{\mu \nu} \gamma_{\alpha} q^{\alpha}\right] F_{1}(\xi, \eta) } \\
& -\gamma_{\mu} F_{1, \nu}(\xi, \eta)-\gamma_{\nu} F_{1, \mu}(\xi, \eta)+\gamma_{\alpha} q^{\alpha} F_{2, \mu \nu}(\xi, \eta) \\
\Gamma_{\mu \nu}^{F 5}\left(q, p_{+}, p_{-}\right)= & i \gamma_{5} \varepsilon_{\mu \nu \lambda \sigma}\left[q^{\lambda} \gamma^{\sigma} F_{1}^{5}(\xi, \eta)+q^{\lambda} \gamma_{\alpha} q^{\alpha} F_{2}^{\sigma, 5}(\xi, \eta)\right] \\
\Gamma_{\mu \nu}^{G}\left(q, p_{+}, p_{-}\right)= & {\left[q_{\mu} \sigma_{\nu \alpha} p_{-}^{\alpha}+q_{\nu} \sigma_{\mu \alpha} p_{-}^{\alpha}-g_{\mu \nu} \sigma_{\beta \alpha} p_{-}^{\alpha} q^{\beta}\right] G_{1}(\xi, \eta) } \\
& -\sigma_{\mu \alpha} p_{-}^{\alpha} G_{1, \nu}(\xi, \eta)-\sigma_{\nu \alpha} p_{-}^{\alpha} G_{1, \mu}(\xi, \eta) p_{-}^{\alpha}+\sigma_{\beta \alpha} p_{-}^{\alpha} q^{\beta} G_{2, \mu \nu}(\xi, \eta) \\
\Gamma_{\mu \nu}^{G 5}\left(q, p_{+}, p_{-}\right)= & i \gamma_{5} \varepsilon_{\mu \nu \lambda \sigma}\left[q^{\lambda} \sigma^{\sigma \alpha} p_{-\alpha} G_{1}^{5}(\xi, \eta)+q^{\lambda} \sigma^{\alpha \beta} q_{\alpha} p_{-\beta} G_{2}^{\sigma, 5}(\xi, \eta)\right] .
\end{aligned}
$$

The different amplitude functions are given by

$$
\begin{aligned}
H_{1}(\xi, \eta) & =\int D z \frac{1}{Q^{2}+i \varepsilon} H\left(z_{+}, z_{-}\right) \\
H_{k}^{\sigma}(\xi, \eta) & =\int D z \frac{p_{+}^{\sigma} z_{+}+p_{-}^{\sigma} z_{-}}{\left(Q^{2}+i \varepsilon\right)^{k}} H\left(z_{+}, z_{-}\right)=\int D z \frac{p_{+}^{\sigma} t+\pi_{\sigma} z_{-}}{\left(Q^{2}+i \varepsilon\right)^{k}} H\left(z_{+}, z_{-}\right) \\
H_{2, \mu \nu}(\xi, \eta) & =\int D z \frac{1}{\left(Q^{2}+i \varepsilon\right)^{2}}\left[-p_{z \mu} p_{z \nu}+q_{\nu} p_{z \mu}+q_{\mu} p_{z \nu}-g_{\mu \nu} q \cdot p_{z}\right] H\left(z_{+}, z_{-}\right) \\
& =\int D z \frac{1}{\left(Q^{2}+i \varepsilon\right)^{2}}\left[-p_{+\mu} p_{+\nu} t^{2}+\left(q_{\nu} p_{+\mu}+q_{\mu} p_{+\nu}\right) t-g_{\mu \nu} q \cdot p_{z}\right. \\
\left.-\pi_{\mu} \pi_{\nu} z_{-}^{2}+\left(q_{\nu} \pi_{\mu}+q_{\mu} \pi_{\nu}\right) z_{-}+\left(p_{+\nu} \pi_{\mu}+p_{+\mu} \pi_{\nu}\right) t z_{-}\right] & \times H\left(z_{+}, z_{-}\right),
\end{aligned}
$$

where

$$
\begin{aligned}
t & =z_{+}+\eta z_{-} \\
\pi_{\sigma} & =p_{-\sigma}-\eta p_{+\sigma}
\end{aligned}
$$

and $q \cdot p_{z}=q \cdot p_{+} t$. Here $H$ denotes either $F$ or $G$. The amplitude functions in Eqs. 2.412.44) depend on the scaling variables $\xi$ and $\eta$. In the limit of forward scattering $(\eta \rightarrow 0, \xi \rightarrow$ $\left.x_{B}\right)$ the structure functions $F_{1,2}\left(x_{B}\right)$ and $g_{1,2}\left(x_{B}\right)$ occur as the limit of the amplitude functions of respective helicity projections from the DIRAC-type terms. The relations between these amplitude functions are derived in Section 6 . 


\section{Kinematic Relations}

For convenience we construct the helicity basis for the photons $\gamma_{1}^{*}$ and $\gamma_{2}^{(*)}$ in the Breit-frame

$$
\begin{aligned}
p_{+} & =p_{1}+p_{2}=\left(2 E_{p} ; \overrightarrow{0}\right) \\
-p_{-} & =p_{1}-p_{2}=(0 ; 2 \vec{p})=\left(0 ; 0,0,2 p_{3}\right) \\
q & =\frac{1}{2}\left(q_{1}+q_{2}\right)=\left(q_{0} ; q_{1}, 0, q_{3}\right) .
\end{aligned}
$$

The matrix elements

$$
\mathrm{T}_{k l}=\varepsilon_{2, k}^{\mu} T_{\mu \nu} \varepsilon_{1, l}^{\nu}, \quad k, l \in\{0,1,2,3\}
$$

are independent of the reference frame.

In the generalized Bjorken region we classify the various contributions to $\mathrm{T}_{k l}$ by their $\nu_{-}$ dependence, where the leading terms are kept respectively. There the kinematic invariants are given by

$$
\begin{aligned}
q_{1} \cdot q_{1} & =-\nu(\xi-\eta) \\
q_{2} \cdot q_{2} & =-\nu(\xi+\eta) \\
q \cdot p_{+} & =\nu \\
q \cdot p_{-} & =\eta \nu \\
q \cdot q & =-\xi \nu \\
q \cdot p_{z} & =q^{2}-Q^{2}=\left(z_{+}+z_{-} \eta\right) \nu \equiv t \nu \\
p_{+}^{2} & \approx p_{-}^{2} \approx p_{+} p_{-} \approx 0 .
\end{aligned}
$$

To define the helicity basis we introduce the two reference vectors

$$
\begin{aligned}
& n_{0}=(1 ; 0,0,0) \\
& n_{2}=(0 ; 0,1,0) .
\end{aligned}
$$

The polarization vectors of the photons $\gamma_{1}^{*}$ and $\gamma_{2}^{*}$ are then given by

$$
\begin{aligned}
\varepsilon_{0 \mu}^{(1)} & =\frac{q_{1 \mu}}{\sqrt{\left|q_{1}^{2}\right|}}=\frac{q_{1 \mu}}{\nu^{1 / 2}} \frac{1}{\sqrt{|\xi-\eta|}} \\
\varepsilon_{0 \mu}^{(2)} & =\frac{q_{2 \mu}}{\sqrt{\left|q_{2}^{2}\right|}}=\frac{q_{2 \mu}}{\nu^{1 / 2}} \frac{1}{\sqrt{|\xi+\eta|}} \\
\varepsilon_{1 \mu}^{(i)} & =n_{2 \mu} \\
\varepsilon_{2 \mu}^{(i)} & =\frac{1}{N_{2 i}} \varepsilon_{\mu \alpha \beta \gamma} n_{0}^{\alpha} n_{2}^{\beta} q_{i}^{\gamma} \\
\varepsilon_{3 \mu}^{(i)} & =\frac{1}{N_{3 i}}\left[q_{i \mu} q_{i} . n_{0}-n_{0 \mu} q_{i} . q_{i}\right]
\end{aligned}
$$

with $i=1,2$ and

$$
\begin{aligned}
& N_{21}=\frac{\nu}{\mu} \sqrt{\left|1+\frac{\mu^{2}}{\nu}(\xi-\eta)\right|} \\
& N_{22}=\frac{\nu}{\mu} \sqrt{\left|1+\frac{\mu^{2}}{\nu}(\xi+\eta)\right|}
\end{aligned}
$$




$$
\begin{aligned}
& N_{31}=\frac{\nu^{3 / 2}}{\mu} \sqrt{|\xi-\eta|} \sqrt{\left|1+\frac{\mu^{2}}{\nu}(\xi-\eta)\right|} \\
& N_{32}=\frac{\nu^{3 / 2}}{\mu} \sqrt{|\xi+\eta|} \sqrt{\left|1+\frac{\mu^{2}}{\nu}(\xi+\eta)\right|},
\end{aligned}
$$

with $\mu^{2}=p_{+}^{2}$.

With respect to the vector $q_{2}$, Eq. (3.4), the above notation applies for space- or time-like vectors, for which $|\xi \pm \eta| \neq 0$ and all the above terms are regular. The polarization vectors obey

$$
\varepsilon_{k \mu}^{(i)} \cdot \varepsilon_{l}^{(i) \mu}=s_{k} \delta_{k l}
$$

with $s_{k}=-1$ for $k=0,1,2$ and $s_{k}=+1$ for $k=3$ if both vectors are space-like and analogous relations if $q_{2}$ is time-like. $\varepsilon_{1,2 \mu}^{(i)}$ are the transversal and $\varepsilon_{3 \mu}^{(i)}$ is the longitudinal polarization vector of the photon $\gamma_{i}^{*}$.

As will be shown below the hadronic mass scale $\mu^{2}=p_{+}^{2}$ occurring as a normalization parameter cancels in the helicity projections of the Compton amplitude in leading order in $1 / \nu$, cf. Section 5 .

For the limit to the forward case and the understanding of the results in the limit $\mu^{2} \ll \nu$ it is useful to rewrite the polarization vectors 3.12 3.15) as

$$
\begin{aligned}
\varepsilon_{0 \rho}^{(1(2))} & =\frac{1}{\sqrt{|\xi|}}\left[\frac{q_{\rho}}{\nu^{1 / 2}} \pm \frac{p_{-\rho}}{2 \nu^{1 / 2}}\right] \frac{1}{\sqrt{|1 \mp \eta / \xi|}} \\
\varepsilon_{1 \rho}^{(1(2))} & =n_{2 \rho} \\
\varepsilon_{2 \rho}^{(1(2))} & =\frac{\mu}{\nu}\left|1-\frac{\mu^{2}}{2 \nu}(\xi \mp \eta)\right| \varepsilon_{\rho \alpha \beta \gamma} n_{0}^{\alpha} n_{2}^{\beta}\left(q^{\gamma} \pm \frac{1}{2} p_{-}^{\gamma}\right) \\
\varepsilon_{3 \rho}^{(1(2))} & =\frac{1}{\nu^{1 / 2}} \frac{1}{\sqrt{|\xi|}}\left|1-\frac{\mu^{2}}{2 \nu}(\xi \mp \eta)\right|\left[q_{\rho} \pm \frac{1}{2} p_{-\rho}+\mu n_{0 \rho}(\xi \mp \eta)\right] \frac{1}{\sqrt{|1 \mp \eta / \xi|}} .
\end{aligned}
$$

In the limit $p_{-\rho}, \eta \rightarrow 0$ the polarization vectors of the first and the second photon become identical. On the other hand, in the limit that terms of $O\left(\mu^{2}\right)$ can be neglected against terms of $O(\nu)$ the polarization vectors $\varepsilon_{3 \rho}^{(i)}$ and $\varepsilon_{0 \rho}^{(i)}$ become identical for $i=1,2$. The latter aspect induces that current conservation for the twist-2 contributions, cf. section 4 , enforces that helicity projections in the direction of the longitudinal polarization vectors vanish in the generalized Bjorken region in lowest order QCD.

If $q_{2}$ is light-like the respective set of polarization vectors reads

$$
\begin{aligned}
\varepsilon_{0 \mu}^{(2)} & =\frac{1}{\sqrt{2} q_{0}^{(2)}} q_{\mu}=\frac{1}{\sqrt{2} q_{0}^{(2)}}\left(q_{0}, \overrightarrow{q_{2}}\right) \\
\varepsilon_{1 \mu}^{(2)} & =n_{2 \mu} \\
\varepsilon_{2 \mu}^{(2)} & =\frac{1}{q_{0}^{(2)}} \varepsilon_{\mu \alpha \beta \gamma} n_{0}^{\alpha} n_{2}^{\beta} q_{2}^{\gamma},
\end{aligned}
$$

with

$$
q_{0}^{(2)}=\frac{\nu}{\mu}
$$


A fourth linearly independent vector associated to the above set is

$$
\widetilde{\varepsilon}_{0 \mu}^{(2)}=\frac{1}{\sqrt{2} q_{0}^{(2)}}\left(q_{0},-\vec{q}_{2}\right)
$$

with

$$
\widetilde{\varepsilon}_{0 \mu}^{(2)}+\varepsilon_{0 \mu}^{(2)}=\sqrt{2} n_{0 \mu} .
$$

The vectors $\widetilde{\varepsilon}_{0 \mu}^{(2)}, \varepsilon_{0 \mu}^{(2)}, \varepsilon_{1 \mu}^{(2)}$ and $\varepsilon_{2 \mu}^{(2)}$ span the Minkowski space. For later use we also note that the vectors

$$
p_{+}^{\mu}, p_{-}^{\mu}, q^{\mu} \quad \text { and } \quad n_{2}^{\mu}
$$

span the Minkowski space for the general case of non-forward scattering, excluding special cases as forward scattering $p_{-}=0$ and vacuum-meson transition $p_{+}=p_{-}$.

\section{Current Conservation}

The conservation of the electromagnetic current

$$
\partial_{\mu}^{x} J^{\mu}(x)=0
$$

implies for the Compton amplitude

$$
\begin{aligned}
T_{\mu \nu}\left(p_{+}, p_{-}, q\right) & =i \int d^{4} e^{-i q_{2} x}\left\langle p_{2}, S_{2}\right| R T\left(J_{\mu}(0) J_{\nu}(x)\left|p_{1}, S_{1}\right\rangle\right. \\
& =i \int d^{4} e^{-i q_{1} x}\left\langle p_{2}, S_{2}\right| R T\left(J_{\mu}(-x) J_{\nu}(0)\left|p_{1}, S_{1}\right\rangle\right.
\end{aligned}
$$

the relations

$$
q_{2}^{\mu} T_{\mu \nu}=T_{\mu \nu} q_{1}^{\nu}=0 .
$$

Expanding the Compton amplitude according to the (non-local) operator product expansion for deep inelastic non-forward scattering in the generalized Bjorken region the terms obtained forming the matrix elements (2.32) are not necessarily yet the twist -2 contributions only. The explicit calculation shows, that still terms of the order $\left(\mu^{2} / \nu\right)^{1 / 2+k}, k \geq 0$ are contained. These terms are of higher twist and vanish for $\nu \rightarrow \infty$ if compared to the leading twist -2 contributions. To prove the current conservation for the twist -2 contributions to the Compton amplitude these terms have to be dealt with in common with the respective higher twist contributions resulting from the operator matrix elements of the higher twist operators.

We are firstly considering the helicity projections of the Compton amplitude onto the states $\varepsilon_{0 \mu}^{(2)}$ and $\varepsilon_{0 \nu}^{(1)}$, respectively, if $q_{2}$ is either space- or time-like. The remaining index is contracted by all the four helicity vectors. As each of the sets of four helicity vectors corresponding to the virtual photons $\gamma_{1,2}^{*}$ spans the complete Minkowski-space, vanishing of the projections in all components proves the current conservation for the twist-2 contributions. We list the individual projections for the contributions to the different distribution functions $F, F_{5}, G$ and $G_{5}$ separately.

$$
\mathrm{T}_{00}^{F}=-\frac{2}{\nu} \bar{u}\left(p_{2}, S_{2}\right) \gamma_{\mu} q^{\mu} u\left(p_{1}, S_{1}\right) \frac{1}{\sqrt{\left|\xi^{2}-\eta^{2}\right|}} \int D z F\left(z_{+}, z_{-}\right)
$$




$$
\begin{aligned}
& \mathrm{T}_{01}^{F}, \mathrm{~T}_{10}^{F}, \mathrm{~T}_{02}^{F}, \mathrm{~T}_{20}^{F}=O\left(\frac{1}{\sqrt{\nu}}\right) \\
& \mathrm{T}_{03}^{F}=-\frac{2}{\nu} \bar{u}\left(p_{2}, S_{2}\right) \gamma_{\mu} q^{\mu} u\left(p_{1}, S_{1}\right) \frac{1}{|\xi-\eta| \sqrt{\left|\xi^{2}-\eta^{2}\right|}} \int D z F\left(z_{+}, z_{-}\right) \\
& \mathrm{T}_{30}^{F}=-\frac{2}{\nu} \bar{u}\left(p_{2}, S_{2}\right) \gamma_{\mu} q^{\mu} u\left(p_{1}, S_{1}\right) \frac{1}{|\xi+\eta| \sqrt{\left|\xi^{2}-\eta^{2}\right|}} \int D z F\left(z_{+}, z_{-}\right) \\
& \mathrm{T}_{00}^{F 5}=0 \\
& \mathrm{~T}_{01}^{F 5}, \mathrm{~T}_{10}^{F 5}, \mathrm{~T}_{02}^{F 5}, \mathrm{~T}_{20}^{F 5}=O\left(\frac{1}{\sqrt{\nu}}\right) \\
& \mathrm{T}_{03}^{F 5}, \mathrm{~T}_{30}^{F 5}=O\left(\frac{1}{\nu}\right) \\
& \mathrm{T}_{00}^{G}=-\frac{2}{\nu} \bar{u}\left(p_{2}, S_{2}\right) \gamma_{\mu} q^{\mu} u\left(p_{1}, S_{1}\right) \frac{1}{\sqrt{\left|\xi^{2}-\eta^{2}\right|}} \int D z G\left(z_{+}, z_{-}\right) \\
& \mathrm{T}_{01}^{G}, \mathrm{~T}_{10}^{G}, \mathrm{~T}_{02}^{G}, \mathrm{~T}_{20}^{G}=O\left(\frac{1}{\sqrt{\nu}}\right) \\
& \mathrm{T}_{03}^{G}=-\frac{2}{\nu} \bar{u}\left(p_{2}, S_{2}\right) \sigma_{\beta \alpha} p_{-}^{\alpha} q^{\beta} u\left(p_{1}, S_{1}\right) \frac{1}{|\xi-\eta| \sqrt{\left|\xi^{2}-\eta^{2}\right|}} \int D z G\left(z_{+}, z_{-}\right) \\
& \mathrm{T}_{30}^{G}=-\frac{2}{\nu} \bar{u}\left(p_{2}, S_{2}\right) \sigma_{\beta \alpha} p_{-}^{\alpha} q^{\beta} u\left(p_{1}, S_{1}\right) \frac{1}{|\xi+\eta| \sqrt{\left|\xi^{2}-\eta^{2}\right|}} \int D z G\left(z_{+}, z_{-}\right) \\
& \mathrm{T}_{00}^{G 5}=0 \\
& \mathrm{~T}_{01}^{G 5}, \mathrm{~T}_{10}^{G 5}, \mathrm{~T}_{02}^{G 5}, \mathrm{~T}_{20}^{G 5}=O\left(\frac{1}{\sqrt{\nu}}\right) \\
& \mathrm{T}_{03}^{G 5}, \mathrm{~T}_{30}^{G 5}=O\left(\frac{1}{\nu}\right) \text {. }
\end{aligned}
$$

Note that

$$
\begin{aligned}
\bar{u}\left(p_{2}, S_{2}\right) \gamma_{\mu} q^{\mu} u\left(p_{1}, S_{1}\right) & \propto \nu \\
\varepsilon_{\alpha, \beta, \gamma, \delta} p_{ \pm}^{\gamma} q^{\delta} & \propto \nu,
\end{aligned}
$$

etc. The contributions to $\mathrm{T}_{k l}^{H(5)}$ of $O(1 / \sqrt{\nu})$ or $O(1 / \nu)$ are of higher twist since the expectation values of the twist -2 operators are multiplied by a further mass ratio, cf. [17]. Since the integrals

$$
\int D z H\left(z_{+}, z_{-}\right)=\int_{-1}^{+1} d z_{+} \int_{-1+\left|z_{+}\right|}^{+1-\left|z_{+}\right|} H\left(z_{+}, z_{-}\right)=0
$$

for $H=F, G$ vanish, all projections Eqs. (4.4 4.17) vanish in the Bjorken limit proving current conservation for the twist-2 contributions in the generalized Bjorken region.

In the case of forward scattering the evaluation of the current-current operator in terms of the twist-2 operators current conservation is easily obtained, cf. Ref. [7]. In the limit $p_{-}, \eta \rightarrow 0$ the above expressions (4.4 4.17) vanish for all values of $\nu$ by virtue of Eq. (4.19). Unlike the forward case which is characterized by only two kinematic vectors $q$ and $p$, the presence of the vector $p_{-}$ 
in the non-forward case allows for twist-3 terms also for spin-averaged matrix elements. These are of $O(1 / \sqrt{\nu})$ relative to the twist-2 contributions.

The above conclusions hold analogously if $q_{2}$ is a light-like vector. Here the polarization vectors Eqs. 3.22 3.24, 3.26 have to be used.

\section{The Helicity Projections of the Compton Amplitude}

In the following we calculate the helicity projections of the twist-2 contributions to the Compton amplitude (2.40) representing the matrices $\Gamma_{\mu \nu}^{O}(2.41-2.44)$ in the generalized Bjorken region. The unpolarized DIRAC-type terms are :

$$
\begin{aligned}
\mathrm{T}_{11}^{F} & =2 \bar{u}\left(p_{2}, S_{2}\right) \gamma_{\mu} q^{\mu} u\left(p_{1}, S_{1}\right)\left[F_{1}(\xi, \eta)+\varepsilon_{1}^{(2) \mu} \varepsilon_{1}^{(1) \nu} F_{2, \mu \nu}(\xi, \eta)\right] \\
\mathrm{T}_{22}^{F} & =2 \bar{u}\left(p_{2}, S_{2}\right) \gamma_{\mu} q^{\mu} u\left(p_{1}, S_{1}\right)\left[F_{1}(\xi, \eta)+\varepsilon_{2}^{(2) \mu} \varepsilon_{2}^{(1) \nu} F_{2, \mu \nu}(\xi, \eta)\right] \\
\mathrm{T}_{k l}^{F} & \propto\left(\frac{1}{\nu}\right)^{1 / 2+n} \quad \text { for the other projections } k, l \in\{1,2,3\} \text { and } n \geq 0,
\end{aligned}
$$

with

$$
\varepsilon_{1}^{(2) \mu} \varepsilon_{1}^{(1) \nu} F_{2 \mu \nu}(\xi, \eta)=\varepsilon_{2}^{(2) \mu} \varepsilon_{2}^{(1) \nu} F_{2 \mu \nu}(\xi, \eta)=\int D z \frac{q \cdot p_{z}}{\left(Q^{2}+i \varepsilon\right)^{2}} F\left(z_{+}, z_{-}\right)
$$

leading to

$$
\mathrm{T}_{11}^{F}=\mathrm{T}_{22}^{F} \text {. }
$$

Correspondingly the polarized DIRAC-type terms yield :

$$
\begin{gathered}
\mathrm{T}_{12}^{F 5}=-\mathrm{T}_{21}^{F 5} \\
\mathrm{~T}_{k l}^{F 5} \propto\left(\frac{1}{\nu}\right)^{1 / 2+n} \quad \text { for the other projections } k, l \in\{1,2,3\} \text { and } n \geq 0 .
\end{gathered}
$$

We define the vector

$$
S_{21}^{\sigma}:=-\frac{1}{2} \bar{u}\left(p_{2}, S_{2}\right) \gamma_{5} \gamma^{\sigma} u\left(p_{1}, S_{1}\right)
$$

In the case of forward scattering, $\left(p_{2}, S_{2}\right) \rightarrow\left(p_{1}, S_{1}\right), S_{21}^{\sigma}$ denotes the nucleon spin vector $S^{\sigma}$. $\mathrm{T}_{12}^{F 5}$ is given by

$$
\mathrm{T}_{12}^{F 5}=i \varepsilon^{\mu \lambda \nu \sigma} \varepsilon_{1 \mu}^{(2)} \varepsilon_{2 \nu}^{(1)} \int D z \frac{q_{\lambda}}{Q^{2}+i \varepsilon}\left[S_{21, \sigma}+\frac{q \cdot S_{21}}{Q^{2}+i \varepsilon} p_{z \sigma}\right] F_{5}\left(z_{+}, z_{-}\right) .
$$

A similar structure is obtained for the PAULI-type terms. The unpolarized contributions read

$$
\begin{aligned}
\mathrm{T}_{11}^{G} & =2 \bar{u}\left(p_{2}, S_{2}\right) \sigma_{\alpha \beta} q^{\alpha} p_{-}^{\beta} u\left(p_{1}, S_{1}\right)\left[G_{1}(\xi, \eta)+\varepsilon_{1}^{(2) \mu} \varepsilon_{1}^{(1) \nu} G_{2, \mu \nu}(\xi, \eta)\right] \\
\mathrm{T}_{22}^{G} & =2 \bar{u}\left(p_{2}, S_{2}\right) \sigma_{\alpha \beta} q^{\alpha} p_{-}^{\beta} u\left(p_{1}, S_{1}\right)\left[G_{1}(\xi, \eta)+\varepsilon_{2}^{(2) \mu} \varepsilon_{2}^{(1) \nu} G_{2, \mu \nu}(\xi, \eta)\right] \\
\mathrm{T}_{k l}^{G} & \propto\left(\frac{1}{\nu}\right)^{1 / 2+n} \quad \text { for the other projections } k, l \in\{1,2,3\} \text { and } n \geq 0,
\end{aligned}
$$

\footnotetext{
${ }^{5}$ Here and in the following we will use the terms unpolarized and polarized for the symmetric and anti-symmetric contributions to the Compton amplitude, respectively. In the spin-averaged case the anti-symmetric terms cancel.
} 
resulting into

$$
\mathrm{T}_{11}^{G}=\mathrm{T}_{22}^{G} .
$$

Here the tensor $G_{2, \mu \nu}$ is obtained from Eq. (5.4) substituting $F$ into $G$.

The polarized PAULI-type terms obey:

$$
\begin{gathered}
\mathrm{T}_{12}^{G 5}=-\mathrm{T}_{21}^{G 5} \\
\mathrm{~T}_{k l}^{G 5} \propto\left(\frac{1}{\nu}\right)^{1 / 2+n} \quad \text { for the other projections } k, l \in\{1,2,3\} \text { and } n \geq 0,
\end{gathered}
$$

with

$$
\mathrm{T}_{12}^{G 5}=i \varepsilon^{\mu \lambda \nu \sigma} \varepsilon_{1}^{(2) \mu} \varepsilon_{2}^{(1) \nu} \int D z \frac{q_{\lambda}}{Q^{2}+i \varepsilon}\left[\Sigma_{21, \sigma}+\frac{q \cdot \Sigma_{21}}{Q^{2}+i \varepsilon} p_{z \sigma}\right] G_{5}\left(z_{+}, z_{-}\right),
$$

and

$$
\Sigma_{21}^{\sigma}:=-\frac{1}{2} \bar{u}\left(p_{2}, S_{2}\right) \gamma_{5} \sigma^{\sigma \alpha} p_{-\alpha} u\left(p_{1}, S_{1}\right) .
$$

A comparison of the structure of the projections $\mathbf{T}_{12(21)}^{F 5, G 5}$ with the corresponding tensor structure for forward scattering, cf. [12, [], shows that the two contributions are related to terms containing the following contributions of structure functions

$$
\begin{aligned}
\propto q_{\lambda} S_{\sigma, 21} & \rightarrow g_{1}\left(x_{B}\right)+g_{2}\left(x_{B}\right) \\
\propto q_{\lambda} p_{z \sigma} & \rightarrow g_{2}\left(x_{B}\right) .
\end{aligned}
$$

In summary, the non-forward Compton amplitude at the level of the twist-2 contributions in lowest order QCD in the generalized Bjorken region is described by two helicity states of both virtual photons. The unpolarized and polarized DIRAC- and PAULI-terms are related to two non-perturbative amplitude functions respectively.

\section{The Integral Relations}

The amplitude functions describing the twist-2 contributions to the virtual Compton amplitude in the generalized Bjorken region obey relations to which we turn now.

\subsection{Unpolarized Contributions}

For the unpolarized contributions to the Compton amplitude the relations

$$
\mathrm{T}_{11}^{F, G}=\mathrm{T}_{22}^{F, G}
$$

hold for the diagonal helicity projections. All other projections vanish at the level of the twist-2 contributions in lowest order QCD in the generalized Bjorken region. Although Eq. (6.1) holds, the partonic interpretation of $\mathrm{T}_{11}^{F, G}$ and $\mathbf{T}_{22}^{F, G}$ for non-forward scattering as given in the case of forward scattering by

$$
F_{2}\left(x_{B}\right)=2 x F_{1}\left(x_{B}\right) \equiv \sum_{q} e_{q}^{2} x\left[q\left(x_{B}\right)+\bar{q}\left(x_{B}\right)\right]
$$


has still to be clarified since the representations (5.1, 5.2, 5.10, 5.11) contain yet two types of $D z$-integrals (2.46,5.4),

$$
\begin{aligned}
\mathrm{H}_{1}(\xi, \eta) & =\int D z \frac{\nu}{Q^{2}+i \varepsilon} H\left(z_{+}, z_{-}\right)=-\int D z \frac{H\left(z_{+}, z_{-}\right)}{\xi+t-i \varepsilon} \\
\mathrm{H}_{2}(\xi, \eta) & =\int D z \frac{\nu q \cdot p_{z}}{\left(Q^{2}+i \varepsilon\right)^{2}} H\left(z_{+}, z_{-}\right)=\int D z \frac{t H\left(z_{+}, z_{-}\right)}{(\xi+t-i \varepsilon)^{2}} .
\end{aligned}
$$

Changing the integration variables to $\left(t, z_{-}\right)$the integration over $z_{-}$can be performed,

$$
\begin{aligned}
\widehat{H}(t, \eta) & =\int_{z_{-}^{\min }}^{z_{-}^{\max }} d z_{-} H\left(t-\eta z_{-}, z_{-}\right)=\int_{0}^{1} \frac{d \lambda}{\lambda^{2}} \int_{z_{-}^{\min }}^{z_{-}^{\max }} d z_{-} h\left(\frac{t}{\lambda}-\eta \frac{z_{-}}{\lambda}, \frac{z_{-}}{\lambda}\right) \\
& =\int_{t}^{\operatorname{sign}(t)} \frac{d z}{z} \hat{h}(z, \eta),
\end{aligned}
$$

with

$$
z_{-}^{\min , \max }=\frac{t \pm 1}{\eta \pm 1}
$$

and

$$
\hat{h}(z, \eta)=\int_{\rho_{\min }}^{\rho_{\max }} d \rho h(z-\eta \rho, \rho)
$$

where $z=t / \lambda, \rho=z_{-} / \lambda$ and $\rho_{\min (\max )}=z_{-, \min (\max )} \cdot(z / t)$. As it will turn out below $\hat{h}(z, \eta)$ denotes an amplitude function on the partonic level. This interpretation does not apply to $\widehat{H}(t, \eta)$.

By partial integration one may rewrite Eq. (6.4). The support of the variable $t$ is $t \epsilon[-1,+1]$. One obtains

$$
\begin{aligned}
\int_{-1}^{+1} d t \frac{t}{(\xi+t-i \varepsilon)^{2}} \int_{t}^{\operatorname{sign}(t)} \frac{d z}{z} \hat{h}(z, \eta)= & \int_{-1}^{+1} d t \frac{1}{\xi+t-i \varepsilon} \int_{t}^{\operatorname{sign}(t)} \frac{d z}{z} \hat{h}(z, \eta) \\
& -\int_{-1}^{+1} d t \frac{1}{\xi+t-i \varepsilon} \hat{h}(t, \eta)
\end{aligned}
$$

which yields

$$
\mathrm{H}_{2}(\xi, \eta)=-\mathrm{H}_{1}(\xi, \eta)-\int_{-1}^{+1} d t \frac{\hat{h}(t, \eta)}{\xi+t-i \varepsilon}
$$

The $z$-integral contributions in Eqs. (5.1,5.2,5.10,5.11) cancel and the helicity projections $T_{11(22)}^{F, G}$ are

$$
\mathrm{T}_{11(22)}^{H}(\xi, \eta) \propto-\int_{-1}^{+1} \frac{\hat{h}(t, \eta)}{\xi+t-i \varepsilon}=-\mathrm{P} \int_{-1}^{+1} d t \frac{\hat{h}(t, \eta)}{\xi+t}-i \pi \hat{h}(\xi, \eta)
$$

which shows that $\mathrm{T}_{11(22)}^{H}$ obeys a 'partonic' description. For the DIRAC-type terms the function $\hat{h}(\xi, \eta)=\hat{f}(\xi, \eta)$ turns into the quark and antiquark densities in the forward limit $\eta \rightarrow 0, \xi \rightarrow x_{B}$. 
To derive the relations on the Lorentz level, we consider Eq. (2.40). The unpolarized part of $T_{\mu \nu}$ contains the following functions :

$$
\begin{aligned}
H_{1}(\xi, \eta)= & -\frac{1}{\nu} \int_{-1}^{+1} d t \frac{1}{\xi+t-i \varepsilon} \hat{\mathrm{H}}(t, \eta) \\
H_{k}^{\sigma}(\xi, \eta)= & \frac{p_{+}^{\sigma}}{(-\nu)^{k}} \int_{-1}^{+1} d t \frac{t}{(\xi+t-i \varepsilon)^{k}} \hat{\mathrm{H}}(t, \eta)+\frac{\pi^{\sigma}}{(-\nu)^{k}} \int_{-1}^{+1} d t \frac{1}{(\xi+t-i \varepsilon)^{2}} \tilde{\mathrm{H}}_{1}(t, \eta) \\
H_{2, \mu \nu}(\xi, \eta)= & \frac{1}{\nu^{2}} \int_{-1}^{+1} d t \frac{1}{(\xi+t-i \varepsilon)^{2}}\left\{\left[-p_{+\mu} p_{+\nu} t^{2}+\left(q_{\nu} p_{+\mu}+q_{\mu} p_{+\nu}\right) t-g_{\mu \nu} q \cdot p_{+} t\right] \hat{\mathrm{H}}(t, \eta)\right. \\
& \left.+\left[\left(p_{+\nu} \pi_{\mu}+p_{+\mu} \pi_{\nu}\right) t+\left(q_{\nu} \pi_{\mu}+q_{\mu} \pi_{\nu}\right)\right] \tilde{\mathrm{H}}_{1}(t, \eta)-\pi_{\mu} \pi_{\nu} \tilde{\mathrm{H}}_{2}(t, \eta)\right\}
\end{aligned}
$$

where $k=1,2$ and

$$
\begin{aligned}
\hat{\mathrm{H}}(t, \eta) & =\int_{t}^{\operatorname{sign}(t)} \frac{d z}{z} \hat{h}(z, \eta) \\
\tilde{\mathrm{H}}_{\mathrm{k}}(t, \eta) & =\int_{t}^{\operatorname{sign}(t)} \frac{d z}{z} \tilde{h}_{k}(z, t, \eta)
\end{aligned}
$$

with

$$
\tilde{h}_{k}(z, t, \eta)=\left(\frac{t}{z}\right)^{k} \int_{\rho_{\min }}^{\rho_{\max }} d \rho \rho^{k} h(z-\eta \rho, \rho)
$$

Note that contractions of the vector $\pi_{\sigma}$ in the above equations with one of the vectors $q^{\mu}, p_{ \pm}^{\mu}$ or $n_{2}^{\mu}$, which span the Minkowski space, yields contributions of at most $O\left(\mu^{2}\right)$ if compared to the large invariants which are of $O(\nu)$. The contributions due to these terms are therefore hadronic mass scale corrections or of higher twist. These terms vanish also both for forward scattering and in the case of vacuum-meson transition $p_{+}=p_{-}, \eta=1$. Due to this we will consider only the terms which do not contain the vectors $\pi_{\sigma}$ in the following. The integrals (6.12, 6.13) may be rewritten by partial integration.

$$
\begin{aligned}
H_{2}^{\sigma}(\xi, \eta)= & \frac{p_{+}^{\sigma}}{\nu^{2}} \int_{-1}^{+1} d t \frac{1}{\xi+t-i \varepsilon}[\hat{\mathrm{H}}(t, \eta)-\hat{h}(t, \eta)]+O\left(\pi^{\sigma}\right) \\
H_{2, \mu \nu}(\xi, \eta)= & \frac{1}{\nu^{2}} \int_{-1}^{+1} d t \frac{1}{\xi+t-i \varepsilon}\left\{-p_{+\mu} p_{+\nu} t[2 \hat{\mathrm{H}}(t, \eta)-\hat{h}(t, \eta)]\right. \\
& \left.\quad+\left[\left(q_{\nu} p_{+\mu}+q_{\mu} p_{+\nu}\right)-g_{\mu \nu} q \cdot p_{+}\right][\hat{\mathrm{H}}(t, \eta)-\hat{h}(t, \eta)]\right\}+O\left(\pi_{\mu(\nu)}\right)
\end{aligned}
$$

Let us define the vector

$$
P_{21}^{\sigma}:=\bar{u}\left(p_{2}, S_{2}\right) \gamma^{\sigma} u\left(p_{1}, S_{1}\right)
$$

with $P_{21}^{\sigma}=p_{+}^{\sigma}$ for forward scattering. For the unpolarized part of $T^{\mu \nu}$, Eq. (2.40), $A^{\mu \nu}$, one obtains

$$
\begin{aligned}
A^{\mu \nu}= & -2 \frac{q \cdot P_{21}}{\nu}\left[g^{\mu \nu}-\frac{q^{\mu} p_{+}^{\nu}+q^{\nu} p_{+}^{\mu}}{q \cdot p_{+}}\right] \int_{-1}^{+1} d t \frac{\mathrm{F}_{1}(t, \eta)}{\xi+t-i \varepsilon} \\
& +\frac{2}{\nu}\left[q^{\mu}\left(P_{21}^{\nu}-p_{+}^{\nu} \frac{q \cdot P_{21}}{\nu}\right)+q^{\nu}\left(P_{21}^{\mu}-p_{+}^{\mu} \frac{q \cdot P_{21}}{\nu}\right)\right] \int_{-1}^{+1} d t \frac{\hat{\mathrm{H}}(t, \eta)}{\xi+t-i \varepsilon}
\end{aligned}
$$




$$
\begin{aligned}
& -\frac{q \cdot P_{21}}{\nu^{2}} p_{+}^{\mu} p_{+}^{\nu} \int_{-1}^{+1} d t \frac{\mathrm{F}_{2}(t, \eta)}{\xi+t-i \varepsilon} \\
& -\frac{2}{\nu}\left[p_{+}^{\mu}\left(p_{21}^{\nu}-p_{+}^{\nu} \frac{q \cdot P_{21}}{\nu}\right)+p_{+}^{\nu}\left(p_{21}^{\mu}-p_{+}^{\mu} \frac{q \cdot P_{21}}{\nu}\right)\right] \int_{-1}^{+1} d t \frac{t \hat{\mathrm{H}}(t, \eta)}{\xi+t-i \varepsilon} .
\end{aligned}
$$

Here we defined

$$
\begin{aligned}
& \mathrm{F}_{1}(t, \eta)=\hat{h}(t, \eta) \\
& \mathrm{F}_{2}(t, \eta)=2 t \hat{h}(t, \eta) .
\end{aligned}
$$

The vector

$$
\Pi^{\mu}=P_{21}^{\mu}-p_{+}^{\mu} \frac{q \cdot P_{21}}{\nu}
$$

in Eq. (6.20), as also the case for the vector $\pi^{\mu}$ above, has contractions with the vectors $q^{\mu}, p_{ \pm}^{\mu}$ and $n_{2}^{\mu}$ which either vanish or are of $O\left(\mu^{2}\right)$ only. Therefore these terms do not contribute at the lowest twist level unlike those due to $\mathrm{F}_{1}(t, \eta)$ and $\mathrm{F}_{2}(t, \eta)$.

$$
\mathrm{F}_{2}(t, \eta)=2 t \mathrm{~F}_{1}(t, \eta)
$$

is the generalization of the CALLAN-GROSS relation for non-forward scattering. These distri-

bution amplitudes have the partonic representation (6.21, 6.22), whereas the other distribution amplitudes of non-leading twist in Eq. (6.20) depend on the function $\hat{\mathrm{H}}(t, \eta)$, which is related to $\hat{h}(t, \eta)$ by the integral Eq. (6.14).

\subsection{Polarized Contributions}

The matrix element $\mathrm{T}_{12}^{H 5}$, Eq. (5.6),

$$
\mathrm{T}_{12}^{H 5}=i \varepsilon^{\mu \lambda \nu \sigma} \varepsilon_{1 \mu}^{(2)} \varepsilon_{2 \nu}^{(1)} B_{\lambda \sigma}
$$

contains the tensor

$$
B_{\lambda \sigma}=\int D z \frac{q_{\lambda}}{Q^{2}+i \varepsilon}\left[S_{21, \sigma}^{H}+\frac{q \cdot S_{21}^{H}}{Q^{2}+i \varepsilon} p_{z \sigma}\right] H_{5}\left(z_{+}, z_{-}\right),
$$

with $S_{21}^{H}=S_{21}\left(\Sigma_{21}\right)$ for $H=F(G)$. It may be rewritten as

$$
B_{\lambda \sigma}=-\frac{1}{\nu} \int D z \frac{q_{\lambda}}{\xi+t-i \varepsilon}\left[S_{21, \sigma}^{H}-\frac{1}{\nu} \frac{t q \cdot S_{21}^{H}}{\xi+t-i \varepsilon} p_{+\sigma}+\frac{1}{\nu} \frac{q \cdot S_{21}^{H}}{\xi+t-i \varepsilon} z_{-} \pi_{\sigma}\right] H_{5}\left(z_{+}, z_{-}\right) .
$$

The latter term in Eq. (6.26) vanishes for forward scattering and in the case of vacuum-meson transition. We perform the integration over $z_{-}$and obtain

$$
\begin{aligned}
B_{\lambda \sigma}= & -\frac{1}{\nu} q_{\lambda} S_{21, \sigma}^{H} \int_{-1}^{+1} d t \frac{1}{\xi+t-i \varepsilon} \int_{t}^{\operatorname{sign}(t)} \frac{d z}{z} \hat{h}_{5}(z, \eta) \\
& -\frac{1}{\nu^{2}} q_{\lambda} p_{+\sigma} q \cdot S_{21}^{H} \int_{-1}^{+1} d t \frac{1}{\xi+t-i \varepsilon}\left[\hat{h}_{5}(t, \eta)-\int_{t}^{\operatorname{sign}(t)} \frac{d z}{z} \hat{h}_{5}(z, \eta)\right] \\
& -\frac{1}{\nu^{2}} q_{\lambda} \pi_{\sigma} q \cdot S_{21}^{H} \int_{-1}^{+1} d t \frac{1}{\xi+t-i \varepsilon} \int_{t}^{\operatorname{sign}(t)} \frac{d z}{z} \widetilde{h}_{5}(z, t, \eta) .
\end{aligned}
$$


Here the distribution amplitude $\widetilde{h}_{5}$ denotes the first moment with respect to $z_{-}$of the function $H_{5}\left(z_{+}, z_{-}\right)$

$$
\widetilde{h}_{5}(z, t, \eta)=\left(\frac{t}{z}\right) \int_{\rho_{\min }}^{\rho_{\max }} d \rho \rho h(z-\eta \rho, \rho)
$$

whereas $\hat{h}(z, \eta)$, Eq. 6.7), is the corresponding 0th moment.

Let us rewrite Eq. (6.27) choosing the notation in analogy to the forward case by

$$
\begin{aligned}
B_{\lambda \sigma} & =-\frac{1}{\nu} q_{\lambda} \int_{-1}^{+1} \frac{d t}{\xi+t-i \varepsilon} \\
& \times\left\{S_{21, \sigma}^{H}\left[\mathrm{G}_{1}(t, \eta)+\mathrm{G}_{2}(t, \eta)\right]+\frac{1}{\nu} p_{+\sigma} q \cdot S_{21}^{H} \mathrm{G}_{2}(t, \eta)+\frac{1}{\nu} \pi_{\sigma} q \cdot S_{21}^{H} \mathrm{G}_{3}(t, \eta)\right\} .
\end{aligned}
$$

One obtains

$$
\begin{aligned}
\mathrm{G}_{1}(t, \eta) & :=\hat{h}_{5}(t, \eta) \\
\mathrm{G}_{2}(t, \eta) & =-\mathrm{G}_{1}(t, \eta)+\int_{t}^{\operatorname{sign}(t)} \frac{d z}{z} \mathrm{G}_{1}(z, \eta) \\
\mathrm{G}_{3}(t, \eta) & :=\int_{t}^{\operatorname{sign}(t)} \frac{d z}{z} \widetilde{h}_{5}(z, t, \eta) .
\end{aligned}
$$

The $t$-integrals in Eq. (6.29) are performed further according to

$$
\int_{-1}^{+1} d t \frac{A(t)}{\xi+t-i \varepsilon}=\mathrm{P} \int_{-1}^{+1} d t \frac{A(t)}{\xi+t}+i \pi A(-\xi)
$$

The polarized non-forward distribution amplitude $\mathrm{G}_{1}(t, \eta)$ has a partonic interpretation as in the unpolarized case Eq. (6.10). Eq. (6.31) is the non-forward generalization of the WANDZURAWILCZEK relation. For the special case of vacuum-meson transition according integral relations were discussed in [19]. In Eq. (6.32) a new distribution amplitude $\mathrm{G}_{3}(t, \eta)$ emerges which is, however, not of twist-2 since the contractions of $\pi_{\sigma}$ with the 4 -momenta of the scattering process are of $O\left(\mu^{2}\right)$ if compared to the other terms which are of $O(\nu)$.

The above results show that the well-known results for forward scattering, the CALLANGross and the WANDZURA-WILCzEK relation are not bound to forward scattering only, but are of more general validity. In particular their derivation does not require to use the optical

theorem. Furthermore, the above relations hold for quite general functions $H_{(5)}\left(z_{+}, z_{-}\right)$. In deriving the above relations assumptions on their complex structure had not to be made. The $O\left(\mu^{2} / \nu\right)$ corrections are associated with new distribution amplitudes, cf. the contributions $\propto \pi_{\sigma}$ or $\Pi_{\sigma}$ in Eqs. (2.46, 2.47, 6.12, 6.13, 6.20, 6.29), which are either representable by integral relations of leading twist distribution amplitudes or are higher moments in $z_{-}$of the functions $H\left(z_{+}, z_{-}\right)$and $H^{5}\left(z_{+}, z_{-}\right)$, respectively.

\subsection{Forward Scattering}

After the above considerations the limit to forward scattering is easily performed. Because the PAULI-type terms vanish linearly with $p_{-}$only the DIRAC-type terms remain. For forward scattering both the functions $\mathrm{F}_{1}(\xi, \eta)$ and $\mathrm{G}_{1}(\xi, \eta)$ are real. The absorptive part of the Compton amplitude, the hadronic tensor $W_{\mu \nu}$, is given by

$$
W_{\mu \nu}=\frac{1}{2 \pi} \operatorname{lm} T_{\mu \nu}
$$


To derive the forward structure functions we rewrite Eqs. (6.20, 6.27) applying the symmetry relations Eqs. (2.29, 2.30) interchanging $\left(z_{+}, z_{-}\right) \leftrightarrow\left(-z_{+},-z_{-}\right)$, i.e. $t \leftrightarrow-t$, respectively. We define the branches of the functions $\mathrm{F}_{1}(t, 0)$ and $\mathrm{G}_{1}(t, 0)$ for $-1 \leq t<0$ and $0<t \leq+1$ as the antiquark and quark distribution function by

$$
\begin{aligned}
& \mathrm{F}_{1}(t, 0)=\sum_{q} e_{q}^{2}[q(t) \theta(t)-\bar{q}(-t) \theta(-t)] \\
& \mathrm{G}_{1}(t, 0)=\sum_{q} e_{q}^{2}[\Delta q(t) \theta(t)+\Delta \bar{q}(-t) \theta(-t)] .
\end{aligned}
$$

For the unpolarized contributions one obtains for forward scattering, $q=q_{1}=q_{2}, p=p_{+} / 2$,

$$
q_{\mu} A^{\mu \nu}=p^{\nu}\left[\int_{-1}^{+1} \frac{2 \xi \mathrm{F}_{1}(t, 0)-\mathrm{F}_{2}(t, 0)}{\xi-t-i \varepsilon}-\int_{-1}^{+1} \frac{2 \xi \mathrm{F}_{1}(t, 0)+\mathrm{F}_{2}(t, 0)}{\xi+t-i \varepsilon}\right]=0 .
$$

Taking the absorptive part yields

$$
\pm 2 \xi \mathrm{F}_{1}( \pm \xi, 0)=\mathrm{F}_{2}( \pm \xi, 0)
$$

The structure functions are now given by

$$
\begin{aligned}
& F_{1}\left(x_{B}\right)=\frac{1}{2}\left[\mathrm{~F}_{1}(\xi, 0)-\mathrm{F}_{1}(-\xi, 0)\right]=\frac{1}{2} \sum_{q} e_{q}^{2}\left[q\left(x_{B}\right)+\bar{q}\left(x_{B}\right)\right], \\
& F_{2}\left(x_{B}\right)=\mathrm{F}_{2}(\xi, 0)+\mathrm{F}_{2}(-\xi, 0),
\end{aligned}
$$

with the Bjorken variable $x_{B}=\lim _{\eta \rightarrow 0} \xi$. The structure functions obey

$$
F_{2}\left(x_{B}\right)=2 x_{B} F_{1}\left(x_{B}\right)
$$

the CALlan-Gross relation [13].

Correspondingly, for the polarized part one obtains

$$
\begin{aligned}
B_{\lambda \sigma}= & -\frac{1}{2 \nu} q_{\lambda} S_{21, \sigma}^{H} \int_{-1}^{+1} d t\left[\frac{\mathrm{G}_{1}(t, 0)+\mathrm{G}_{2}(t, 0)}{\xi+t-i \varepsilon}+\frac{\mathrm{G}_{1}(t, 0)+\mathrm{G}_{2}(t, 0)}{\xi-t-i \varepsilon}\right] \\
& -\frac{1}{2 \nu^{2}} q_{\lambda} p_{+\sigma} q . S_{21}^{H} \int_{-1}^{+1} d t\left[\frac{\mathrm{G}_{2}(t, 0)}{\xi+t-i \varepsilon}+\frac{\mathrm{G}_{2}(t, 0)}{\xi-t-i \varepsilon}\right] .
\end{aligned}
$$

The absorptive part is described by the structure functions $g_{1}\left(x_{B}\right)$ and $g_{2}\left(x_{B}\right)$ with, cf. (6.27),

$$
\begin{aligned}
& g_{1}\left(x_{B}\right)=\frac{1}{2}\left[\mathrm{G}_{1}(\xi, 0)+\mathrm{G}_{1}(-\xi, 0)\right]=\frac{1}{2} \sum_{q} e_{q}^{2}\left[\Delta q\left(x_{B}\right)+\Delta \bar{q}\left(x_{B}\right)\right] \\
& g_{2}\left(x_{B}\right)=-g_{1}\left(x_{B}\right)+\int_{x_{B}}^{1} \frac{d z}{z} g_{1}(z) .
\end{aligned}
$$

Eq. (6.44) is the WANDZURA-WILCZEK relation [14].

\footnotetext{
${ }^{6}$ In the presence of electroweak currents five polarized structure functions contribute on the level of twist-2 and for the quark operators also for twist-3. These structure functions are connected by two further relations for the twist-2 contributions, the Dicus-relation [20] and a relation by BlüMlein and Kochelev [6]. The three twist -3 relations among the respective contributions to the polarized structure functions due to the quark operators at lowest order QCD have been derived in Ref. [7] recently. One of these relations applies to the case of pure electromagnetic interactions.
} 


\section{Conclusions}

We studied the structure of the virtual Compton amplitude for deep-inelastic non-forward scat-

tering $\gamma^{*}+p \rightarrow \gamma^{(*)^{\prime}}+p^{\prime}$ in lowest order in QCD in the massless limit. In the generalized Bjorken region $q \cdot p_{+},-q^{2} \rightarrow \infty$ the twist-2 contributions to the Compton amplitude were calculated using the non-local operator product expansion for general spin states. In this approximation the Compton amplitude consists of an unpolarized and a polarized DIRAC- and PAULI-type amplitude, the latter of which vanishes in the case of forward scattering. The expectation values of the (non-local) twist-2 vector operators in the non-forward case do still contain terms $\propto 1 / \nu^{1 / 2+k}, k \geq 0$, which are contributions of twist-3 and higher to the Compton amplitude. These contributions have to be considered in common with the non-forward expectation values of the higher twist operators. A decomposition of the amplitude was performed with respect to the helicity states of both (virtual) photons. The twist-2 contributions are due to two polarization states only, which are for the unpolarized part $T_{11}$ and $T_{22}$ and for the polarized part $T_{12}$ and $T_{21}$. For the twist- 2 contributions the gauge invariance of the non-local light cone expansion was proven in the non-forward case in the generalized Bjorken region. The relations between the twist-2 contributions of the unpolarized and polarized amplitude functions were derived. They are the non-forward generalizations of the CALlan-Gross and WANDZURA-WiLCZEK relations for unpolarized and polarized deep-inelastic forward scattering. The relations for the DIRAC and PAULI parts are of the same form.

\section{Acknowledgement}

Our thanks are due to B. Geyer for fruitful discussions and U. Gensch for his constant support. Discussions with A. Tkabladze in an early phase of this project are acknowledged. J.B. would like to thank the Institute of Theoretical Physics at Graz University for their kind hospitality. D.R. likes to thank DESY Zeuthen and the Institute of Theoretical Physics at Graz University for the kind hospitality extended to him, in particular to C.B. Lang, H. Mitter, N. Pucker, and W. Schweiger. 


\section{References}

[1] K.G. Wilson, Phys. Rev. 179 (1969) 1699;

R.A. Brandt and G. Preparata, Fortschr. Phys. 18 (1970) 249;

W. Zimmermann, Lect. on Elementary Particle Physics and Quantum Field Theory, Brandeis Summer Inst., Vol. 1, (MIT Press, Cambridge, 1970), p. 395.

[2] Y. Frishman, Ann. Phys. 66 (1971) 373.

[3] D.J. Gross and S.B. Treiman, Phys. Rev. D4 (1971) 1059.

[4] D.J. Gross and F. Wilczek, Phys. Rev. D8 (1973) 3633; D9 (1974) 980;

H. Georgi and D. Politzer, Phys. Rev. D9 (1974) 416;

W. Furmanski and R. Petronzio, Z. Phys. C11 (1982) 293 and references therein;

G. Floratos, P. Lacaze, and C. Kounnas, Phys. Lett. B98 (1980) 437; Nucl. Phys. 192 (1981) 417;

G. Curci, W. Furmanski, and R. Petronzio, Nucl. Phys. B175 (1980) 27;

W. Furmanski and R. Petronzio, Phys. Lett. B97 (1980) 437;

R. Hamberg and W.L. van Neerven, Nucl. Phys. B379 (1992) 143;

E.B. Zijlstra and W.L. van Neerven, Phys. Lett. B273 (1991) 476; Nucl. Phys. B383 (1992) 525 ;

W.L. van Neerven and E.B. Zijlstra, Phys. Lett. B272 (1991) 127;

S. Moch and J. Vermaseren, hep-ph/9912355;

S. Larin, T. van Ritbergen, and. J. Vermaseren, Nucl. Phys. B427 (1994) 41;

S. Larin, P. Nogueira, T. van Ritbergen, and. J. Vermaseren, Nucl. Phys. B492 (1997) 338 ;

Y. Matiounine, J. Smith, and W.L. van Neerven, Phys. Rev. D57 (1998) 6701; D58 (1998) 076002;

G. Altarelli and G. Parisi, Nucl. Phys. B126 (1977) 298 and references therein;

R. Mertig and W.L. van Neerven, Z. Phys. C70 (1996) 637;

W. Vogelsang, Phys. Rev. D54 (1996) 2023;

E.B. Zijlstra and W.L. van Neerven, Phys. Lett. B297 (1992) 377; Nucl. Phys. B417 (1994) 61; E: B426 (1994) 245.

[5] E. Derman, Phys. Rev. D19 (1979) 133.

[6] J. Blümlein and N. Kochelev, Phys. Lett. B381 (1996) 296; Nucl. Phys. B498 (1997) 285.

[7] J. Blümlein and A. Tkabladze, Nucl. Phys. B553 (1999) 427.

[8] T. Braunschweig, B. Geyer, and D. Robaschik, Ann. Phys. (Leipzig) 44 (1987) 407;

I.I. Balitsky and V.M. Braun, Nucl. Phys. B311 (1988/89) 541;

A.V. Radyushkin, Phys. Lett. B385 (1996) 333; Phys. Rev. D56 (1997) 5524;

J. Blümlein, B. Geyer, and D. Robaschik, Phys. Lett. B406 (1997) 161 and Erratum;

I.I. Balitsky and A.V. Radyushkin, Phys. Lett. B413 (1997) 114;

J. Blümlein, B. Geyer, and D. Robaschik, hep-ph/9711405, in : Proc. of the Workshop Deep Inelastic Scattering Off Polarized Targets: Theory Meets Experiment, eds. J. Blümlein et al., (DESY, Hamburg, 1997) DESY 97-200, p. 196.

X. Ji, Phys. Rev. Lett. 78 (1997) 610; Phys. Rev. D55 (1997) 7114; 
L. Mankiewicz, G. Piller, and T. Weigl, Eur. J. Phys. C5 (1998) 119;

X. Ji, J. Phys. G24 (1998) 1181.

[9] H1 collaboration, C. Adloff et al., Phys. Lett. B393 (1997) 452 and references therein.

[10] E143 collaboration, K. Abe et al., Phys. Rev. D58 (1998) 112003;

E155 collaboration, P. Anthony et al., Phys. Lett. B458 (1999) 529.

[11] S.A. Anikin, M.C. Polivanov, and O.I. Zavialov, Fortschr. Physik 27 (1977) 459;

S.A. Anikin and O.I. Zavialov, Ann. Phys. (NY) 116 (1978) 135;

O.I. Zavialov, Renormalized Feynman Diagrams, (Nauka, Moscow, 1979), in Russian;

Renormalized Quantum Field Theory (Kluwer Academic Press, Dordrecht, 1990);

D. Müller, D. Robaschik, B. Geyer, F. Dittes, and J. Hořejši, Fortschr. Phys. 42 (1994) 2.

[12] J. Blümlein, B. Geyer, and D. Robaschik, Nucl. Phys. B560 (1999) 283.

[13] C.G. Callan and D.J. Gross, Phys. Rev. Lett. 22 (1969) 156.

[14] S. Wandzura and F. Wilczek, Phys. Lett. B72 (1977) 95.

[15] W.A. Bardeen and Wu-Ki Tung, Phys. Rev. 173 (1968) 1423;

A. De Rújula and E. De Rafael, Ann. Phys. (NY) 78 (1973) 132;

R. Tarrach, Nuovo Cim. 28A (1975) 409;

D. Drechsel, G. Knöchlein, A.Y. Korchin, A. Metz, and S. Scherer, Phys. Rev. C57 (1998) 941.

[16] B. Geyer, M. Lazar, and D. Robaschik, Nucl. Phys. B559 (1999) 339.

[17] J. Blümlein and W.L. van Neerven, in preparation.

[18] J.L. Miramontes and J. Sánchez Guillén, Z. Phys. C41 (1988) 247.

[19] P. Ball, V.M. Braun, Y. Koike, and K. Tanaka, Nucl. Phys. B529 (1998) 323.

[20] D.A. Dicus, Phys. Rev. D5 (1972) 1367. 